\title{
Efficient Emission from Charge Recombination during the Pulse Radiolysis of Electrochemical Luminescent Substituted Quinolines with Donor-Acceptor Character
}

\author{
Shingo Samori, Michihiro Hara, Sachiko Tojo, Mamoru Fujitsuka, Shu-Wen Yang, ${ }^{\dagger}$ \\ Arumugasamy Elangovan, ${ }^{\dagger}$ Tong-Ing Ho ${ }^{\dagger}$ and Tetsuro Majima* \\ The Institute of Scientific and Industrial Research (SANKEN), Osaka University, Mihogaoka 8-1, \\ Ibaraki, Osaka 567-0047, Japan
}

Received: March 15, 2005; In Final Form: April 15, 2005

\begin{abstract}
Efficient emission from various donor-acceptor quinolines with an ethynyl linkage (PnQ), which are known as efficient electrogenerated chemiluminescent molecules, was observed with time-resolved fluorescence measurement during the pulse radiolysis in benzene. On the basis of the transient absorption and emission measurements, and steady-state measurements, the formation of $\mathbf{P n Q}$ in the singlet excited state can be interpreted by charge recombination between the PnQ radical cation and the PnQ radical anion which are generated initially from the radiolytic reaction in benzene. The strong electronic coupling between the donor and acceptor through conjugation is responsible for the efficient emission during the pulse radiolysis of PnQ in benzene. It is suggested that the positive and negative charges are localized on the donor and acceptor moieties in the radical cation and anion, respectively. This mechanism is reasonably explained by the relationship between the annihilation enthalpy changes and singlet excitation energies of PnQ. The formation of the intramolecular charge transfer state is assumed for PnQ in the singlet excited state with a strong electron donating substituent. The emission from PnQ is suggested to originate from PnQ in the singlet excited state formed from the charge recombination between the PnQ radical cation and the PnQ radical anion during the pulse radiolysis. This is strong evidence for the efficient electrogenerated chemiluminescence of PnQ.
\end{abstract}

\section{Introduction}

In the pulse radiolysis of solutions, the solvent molecules are initially ionized to give hole and electron which react with the solvent molecules. Therefore, the hole or electron can be trapped selectively by the solvent molecules. ${ }^{1}$ For example, the electron is trapped by 1,2-dichloroethane (DCE) through the dissociative electron attachment, while the hole is trapped by solute molecule (M) with a lower oxidation potential to give $\mathbf{M}$ radical cation $\left(\mathbf{M}^{+}\right){ }^{2}$ On the other hand, the hole is trapped by $\mathrm{N}, \mathrm{N}$ dimethylformamide (DMF), while the electron is trapped by $\mathbf{M}$ with a lower reduction potential to give $\mathbf{M}$ radical anion $\left(\mathbf{M}^{\bullet^{-}}\right) .^{3}$

The hole and electron are initially formed in nonpolar solvents, and undergo mainly the fast geminate charge recombination to give the solvent molecules in the excited states. For example, initial products during the pulse radiolysis of benzene $(\mathrm{Bz})$ are solvated electron $\left(\mathrm{e}^{-} \mathrm{s}\right), \mathrm{Bz}$ radical cation $\left(\mathrm{Bz}^{\bullet+}\right)$, and $\mathrm{Bz}$ in the excited state $\left(\mathrm{Bz}^{*}\right)$, which react with $\mathbf{M}$ to give $\mathbf{M}^{\bullet+}$, $\mathbf{M}^{\bullet-}$, and $\mathbf{M}$ in the singlet and triplet excited states $\left(\mathbf{M}^{*}={ }^{1} \mathbf{M}^{*}\right.$ and ${ }^{3} \mathbf{M}^{*}$, respectively) as shown in Scheme $1 .{ }^{4}$ Usually $\mathbf{M}^{*}$ is the main product, while $\mathbf{M}^{\bullet+}$ and $\mathbf{M}^{\bullet-}$ are produced in minor yields because only a small fraction of initially formed coulombcorrelated pairs $\left(\mathrm{Bz}^{\circ} / \mathrm{e}^{-} \mathrm{s}\right)$ is capable of undergoing diffusional escape resulting in the formation of relatively long-lived, homogeneously distributed $\mathbf{M}^{\bullet+}$ and $\mathbf{M}^{\mathbf{0}^{-}}$in a radiolytic process. Therefore, the formation of $\mathbf{M}^{*}$ from the charge recombination

* To whom correspondence should be addressed. Phone: $81+6-6879$ 8495. Fax: 81 +6-6879-8499. E-mail: majima@sanken.osaka-u.ac.jp.

† Present address: Department of Chemistry, Faculty of Science, National Taiwan University, Taipei,Taiwan.
SCHEME 1: Pulse Radiolysis of $\mathrm{M}$ in $\mathrm{Bz}$

$$
\begin{aligned}
& \mathrm{Bz} \rightarrow \mathrm{e}_{\mathrm{s}}^{-}+\mathrm{Bz}^{\bullet+} \\
& \mathrm{Bz}^{\bullet+}+\mathrm{e}_{\mathrm{s}}^{-} \rightarrow \mathrm{Bz}^{*} \\
& \mathrm{e}_{\mathrm{s}}^{-}+\mathbf{M} \rightarrow \mathbf{M}^{\bullet-} \\
& \mathrm{Bz}^{\bullet+}+\mathbf{M} \rightarrow \mathrm{Bz}+\mathbf{M}^{\bullet+} \\
& \mathrm{Bz}^{*}+\mathbf{M} \rightarrow \mathrm{Bz}+\mathbf{M}^{*} \\
& \mathbf{M}^{\bullet+}+\mathbf{M}^{\bullet-} \rightarrow \mathbf{M}^{*}+\mathbf{M}
\end{aligned}
$$

between $\mathbf{M}^{\bullet+}$ and $\mathbf{M}^{\bullet-}$ is a minor process, and no ${ }^{1} \mathbf{M}^{*}$ and only a little ${ }^{3} \mathbf{M}^{*}$ exist at a few tens of nanoseconds after an electron pulse during the pulse radiolysis.

In electrogenerated chemiluminescence (ECL), ${ }^{5} \mathbf{M}^{\bullet+}$ and $\mathbf{M}^{\bullet-}$ are generated from the electrochemical reaction in solution, and the emission mainly results from ${ }^{1} \mathbf{M}^{*}$ and/or the singlet excimer of ${ }^{1} \mathbf{M}^{*}\left({ }^{1} \mathbf{M}_{2} *\right)$, generated directly from the charge recombination between $\mathbf{M}^{\bullet+}$ and $\mathbf{M}^{\mathbf{0}^{-}}$and/or from the triplet-triplet annihilation as shown in Scheme $2 .^{6}$ However, the formation of ${ }^{1} \mathbf{M}^{*}$ and/or ${ }^{1} \mathbf{M}_{2} *$ as the emissive species has not been fully understood.

Recently, design, synthesis, and photophysical properties of the efficient ECL molecules have been developed by various groups. ${ }^{7}$ For example, donor-acceptor type molecules such as (4-phenylethynyl)quinolines and (4-phenylethynyl)isoquinolines, in which the donor and acceptor moieties are linked with an ethynyl group, showed efficient ECL. ${ }^{8}$ It is known that the ECL efficiency is sensitive to the properties of molecules. 
SCHEME 2: Mechanism for the Electrogenerated Chemiluminescence (ECL)

$$
\begin{aligned}
& \mathbf{M}+\mathbf{e}^{-} \rightarrow \mathbf{M}^{\bullet-} \\
& \mathbf{M}-\mathbf{e}^{-} \rightarrow \mathbf{M}^{\bullet+} \\
& \mathbf{M}^{\bullet-}+\mathbf{M}^{\bullet+} \rightarrow{ }^{1} \mathbf{M}^{*}+\mathbf{M},{ }^{3} \mathbf{M}^{*}+\mathbf{M}, \text { and } / \text { or }{ }^{1} \mathbf{M}_{2}{ }^{*} \\
& { }^{3} \mathbf{M}^{*}+{ }^{3} \mathbf{M}^{*} \rightarrow{ }^{1} \mathbf{M}^{*}+\mathbf{M} \text { and/or }{ }^{1} \mathbf{M}_{2}{ }^{*} \\
& { }^{1} \mathbf{M}^{*} \rightarrow \mathbf{M}+h v \\
& { }^{1} \mathbf{M}_{2}{ }^{*} \rightarrow 2 \mathbf{M}+h v^{,}
\end{aligned}
$$

According to the ECL mechanism, if $\mathbf{M}^{\bullet^{+}}$and $\mathbf{M}^{\bullet^{-}}$are produced at sufficient concentrations, ${ }^{1} \mathbf{M}^{*}$ can be generated from the charge recombination between $\mathbf{M}^{\bullet+}$ and $\mathbf{M}^{\bullet-}$ during the pulse radiolysis of the ECL molecules in Bz. However, the formation of $\mathbf{M}^{\bullet+}$ and $\mathbf{M}^{\bullet-}$ is usually a minor process during the pulse radiolysis of various molecules in Bz. We report here, for the first time, observation of the efficient emission from the charge recombination between radical cation and anion of various donor-acceptor type ECL molecules, quinolines and isoquinoline substituted by the 4-( $p$-substituted phenylethynyl) group $(\mathbf{P n Q}=2$ - and 3-( $p$-methoxyphenylethynyl)quinolines (P2Q and P3Q, respectively), 4-( $p$-methoxyphenylethynyl)isoquinoline (P4 $\mathbf{Q} \mathbf{Q})$, and 6-( $p$-substituted phenylethynyl)quinolines $\left.\left(\mathbf{P} 6 \mathbf{Q}_{\mathbf{a}-\mathbf{d}}\right)\right)$ during the pulse radiolysis of $\mathbf{P n Q}$ in Bz. In addition, as the parent unsubstituted molecules of $\mathbf{P n Q}$, quinoline $(\mathbf{Q})$, isoquinoline $\left({ }^{i} \mathbf{Q}\right)$, and several phenylacetylene derivatives $(\mathbf{P A s}=$ phenylacetylene $(\mathbf{P A})$, diphenylacetylene (DPA), and 4-methyldiphenylacetylene $\left.\left(\mathbf{D P A}_{\mathbf{b}}\right)\right)$ were also examined for the emission measurement (Scheme 3). For P2Q, P3Q, and $\mathbf{P} \mathbf{4}^{\mathbf{i}} \mathbf{Q}$, some authors have previously reported the ECL in the electrochemical method. ${ }^{8}$

\section{Experimental Section}

Materials. PnQ were prepared according to the procedure previously described in the literature. ${ }^{8}$ Quinoline $(\mathbf{Q})$, isoquinoline ( $\left.{ }^{\mathbf{i}} \mathbf{Q}\right)$, and phenylacetylene (PA) were purchased from Tokyo Kasei, Aldrich, and Nacali Tesque, respectively, and used as received. Diphenylacetylene (DPA) was purchased from Wako and was recrystallized from ethanol before use. $\mathbf{D P A}_{\mathbf{b}}$ was synthesized from reactions of phenylacetylenylcopper with the methyl-substituted iodobenzene in pyridine according to literature procedures, ${ }^{9}$ and recrystallized from ethanol before use. Spectral grade benzene (Bz) and 1,2-dichloroethane (DCE) were purchased from Nacalai Tesque, and $N, N$-dimethylforma- mide (DMF) was purchased from Wako. They were used as solvent without further purification.

Steady-State Measurements. UV absorption spectra were measured in $\mathrm{Bz}$ by a Shimadzu UV-3100PC UV/visible spectrometer with a transparent rectangular cell made from quartz $\left(1.0 \times 1.0 \times 4.0 \mathrm{~cm}^{3}\right.$, path length $\left.1.0 \mathrm{~cm}\right)$. Fluorescence spectra were measured by a Hitachi 850 spectrofluorometer.

Pulse Radiolysis. The solutions containing samples $(5.0 \mathrm{mM})$ in $\mathrm{Bz}, \mathrm{DCE}$, and DMF were prepared in a rectangular quartz cell $\left(0.5 \times 1.0 \times 4.0 \mathrm{~cm}^{3}\right.$, path length $\left.1.0 \mathrm{~cm}\right)$. These samples were saturated with Ar gas by bubbling for $10 \mathrm{~min}$ at room temperature before irradiation. Pulse radiolysis was performed, as described previously, using an electron pulse $(28 \mathrm{MeV}, 8$ $\mathrm{ns}, 0.7 \mathrm{kGy}$ ) from an L-band linear accelerator at SANKEN, Osaka University. ${ }^{10}$ The monitor light was obtained from a 450-W Xe lamp (Osram, XBO-450), which was operated by a large current pulsed-power supply that was synchronized with the electron pulse. The monitor light was passed through an iris with a diameter of $0.2 \mathrm{~cm}$ and sent into the sample solution with a perpendicular intersection of the electron pulse. Time profiles of the emission and transient absorption were measured with a monochromator (CVI Laser, Digikrom-240) equipped with a PMT (Hamamatsu Photonics, R928) and the signal from the PMT was recorded on a transient digitizer (Tektronix, TDS 580D Digital Phosphor Oscilloscope) and transmitted to a personal computer with a GPIB interface. Time-resolved spectra were measured by a gated-multichannel spectrometer (Unisoku, TSP-601-02). To avoid heating of the sample solution by monitor light, a suitable cutoff filter was used.

Laser Flash Photolysis. To assign the transient species observed during the pulse radiolysis, laser flash photolysis experiments were also carried out by using the third harmonic oscillation $(355 \mathrm{~nm})$ of a nanosecond $\mathrm{Nd}^{3+}$ :YAG laser (Quantel, Brilliant; 5-ns fwhm, $10 \mathrm{~mJ}$ pulse ${ }^{-1}$ ) as an excitation source. The monitor light was obtained from a 450-W Xe lamp (Osram, XBO-450) synchronized with the laser flash. Transient absorption and emission spectra were measured by a streak camera (Hamamatsu Photonics C7700) equipped with a CCD camera (Hamamatsu Photonics C4742-98) or a silicon detector (Hamamatsu Photonics, S5343). Ar- and air-saturated Bz solutions were contained in a transparent rectangular quartz cell $\left(1.0 \times 1.0 \times 4.0 \mathrm{~cm}^{3}\right.$, path length $\left.1.0 \mathrm{~cm}\right)$ at room temperature. The concentration of PnQ was 20-30 $\mu \mathrm{M}$.

Fluorescence Lifetime Measurements. Fluorescence lifetimes were measured by the single photon counting method, using a streakscope (Hamamatsu Photonics, C4334-01) equipped with a polychromator (Acton Research, SpectraPro150). Femtosecond laser pulse was generated with a Ti:sapphire laser (Spectra-Physics, Tsunami 3941-M1BB, fwhm 100 fs) pumped

\section{SCHEME 3}

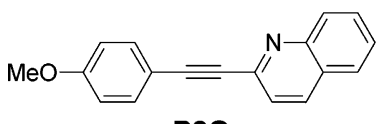

$\mathbf{P} 2 \mathrm{Q}$

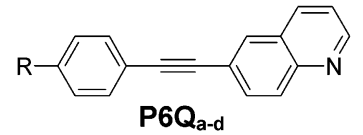

$\mathrm{R}=\mathrm{H}(\mathrm{a}), \mathrm{Me}(\mathrm{b}), \mathrm{OMe}(\mathrm{c}), \mathrm{NEt}_{2}(\mathrm{~d})$

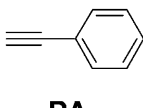

PA
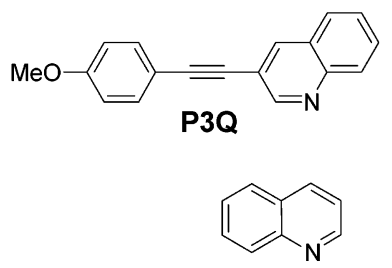

Q

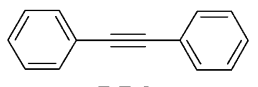

DPA

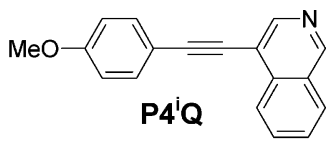<smiles>c1ccc2cnccc2c1</smiles>

'Q

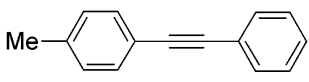

DPA $_{b}$ 
TABLE 1: Photophysical and Electrochemical Properties of PnQ, Q, iQ, and PAs

\begin{tabular}{|c|c|c|c|c|c|c|c|c|c|c|c|}
\hline \multirow[b]{2}{*}{ compd } & \multicolumn{3}{|c|}{ in $\mathrm{CH}_{3} \mathrm{CN}$} & \multicolumn{5}{|c|}{ in $\mathrm{Bz}$} & \multicolumn{3}{|c|}{ in $\mathrm{CH}_{3} \mathrm{CN}$} \\
\hline & $\lambda_{\max }^{\mathrm{Abs}} / \mathrm{nm}$ & $\lambda_{\max }^{\mathrm{Fl}} / \mathrm{nm}$ & $\lambda_{\max }^{\mathrm{ECL}} / \mathrm{nm}$ & $\lambda_{\max }^{\mathrm{Abs}} / \mathrm{nm}$ & $\lambda_{\max }^{\mathrm{Fl}} / \mathrm{nm}$ & $\phi_{\mathrm{fl}}\left(\times 10^{-2}\right)$ & $\tau_{\mathrm{f} /} / \mathrm{ns}$ & $E_{\mathrm{S} 1} / \mathrm{eV}$ & $E_{\mathrm{red}}^{f} / \mathrm{V}$ & $E_{\mathrm{ox}}{ }^{f} / \mathrm{V}$ & $-\Delta H^{\circ} / \mathrm{eV}$ \\
\hline P2Q & $346^{a}$ & $405^{a}$ & $479^{a}$ & 350 & 375 & 15 & 0.23 & 3.31 & $-1.94^{a}$ & $1.71^{a}$ & $3.49^{a}$ \\
\hline P3Q & $342^{a}$ & $406^{a}$ & $b$ & 336 & 373 & 50 & 1.0 & 3.32 & $-1.00^{a}$ & $1.59^{a}$ & $2.43^{a}$ \\
\hline P4iQ & $334^{a}$ & $414^{a}$ & $b$ & 338 & 394 & 62 & 1.1 & 3.15 & $-0.91^{a}$ & $0.58^{a}$ & $1.33^{a}$ \\
\hline$P 6 Q_{a}$ & 338 & 364 & 460 & 313 & 369 & 0.55 & 0.050 & 3.36 & -0.97 & 1.85 & 2.66 \\
\hline$P_{6} Q_{b}$ & 339 & 376 & $450^{c}$ & 315 & 371 & 2.8 & 0.11 & 3.34 & -0.89 & 1.75 & 2.48 \\
\hline$P_{6} Q_{c}$ & 341 & 412 & 475 & 318 & 378 & 44 & 0.85 & 3.28 & -0.90 & 1.50 & 2.24 \\
\hline$P_{6} Q_{d}$ & 366 & 536 & 534 & 372 & 434 & 88 & 1.4 & 2.86 & -0.88 & 0.84 & 1.56 \\
\hline $\mathbf{Q}$ & 270 & 335 & $d$ & 314 & 405 & 0.42 & $d$ & 3.06 & $-2.40^{g}$ & $1.68^{h}$ & 3.92 \\
\hline${ }^{\mathrm{i}} \mathbf{Q}$ & 267 & 336 & $d$ & 319 & 393 & 0.076 & $d$ & 3.16 & $-2.51^{g}$ & $1.55^{h}$ & 3.90 \\
\hline PA & 270 & 317 & $d$ & 280 & 402 & 11 & 2.2 & 3.08 & $-2.81^{i}$ & $1.96^{j}$ & 4.61 \\
\hline DPA & 279 & 331 & $520^{e}$ & 300 & 372 & 3.8 & $d$ & 3.33 & $-2.53-2.8\left(2^{\text {nd }}\right)^{i}$ & $1.52^{k}$ & $3.89,4.16$ \\
\hline $\mathbf{D P A}_{\mathbf{b}}$ & 326 & 420 & $d$ & 279 & 374 & 0.017 & $d$ & 3.32 & $-2.45^{l}$ & $d$ & $d$ \\
\hline
\end{tabular}

${ }^{a}$ Reference 8. ${ }^{b}$ Very weak emission was observed. ${ }^{c}$ Too weak. ${ }^{d}$ Not determined. ${ }^{e}$ Observation of the ECL at this wavelength for DPA is quite unusual. ${ }^{f}$ Redox potentials were estimated vs Ag/Ag ${ }^{+} .{ }^{g}$ Reference $13 \mathrm{a} .{ }^{h}$ Reference $13 \mathrm{~b} .{ }^{i}$ In $N, N$-dimethylformamide, ref $13 \mathrm{c} .{ }^{j}$ Reference $13 \mathrm{~d}$. ${ }^{k}$ Reference $13 \mathrm{e} .{ }^{l}$ In $N, N$-dimethylformamide, ref $13 \mathrm{f}$.

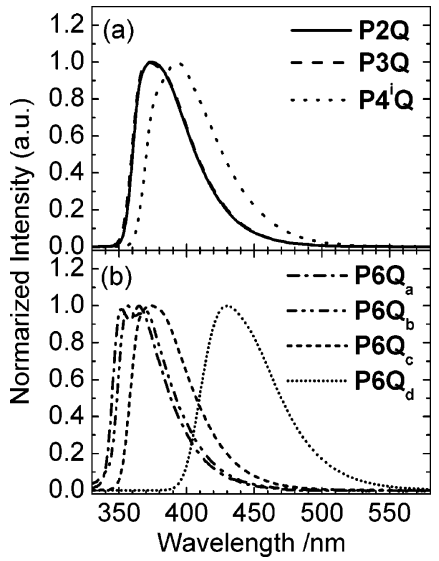

Figure 1. Fluorescence spectra obtained by the steady-state measurement of PnQ in Ar-saturated Bz.

with a diode-pumped solid-state laser (Spectra-Physics, Millennia VIIIs). For the excitation of samples, the output of the Ti:sapphire laser was converted to the third harmonic oscillation $(300 \mathrm{~nm})$ with a harmonic generator (Spectra-Physics, GWU-23FL). In the presence of oxygen, PnQ showed the long lifetime emission corresponds to the "P-type" delayed fluorescence. In this measurement, therefore, all PnQ was prepared in $\mathrm{O}_{2}$-saturated Bz.

\section{Results and Discussion}

Emission Properties of PnQ Together with the Photophysical and Electrochemical Properties. The photophysical properties of $\mathbf{P n Q}, \mathbf{Q},{ }^{\mathbf{i}} \mathbf{Q}$, and $\mathbf{P A s}$ in acetonitrile and $\mathrm{Bz}$ are listed in Table 1, together with the electrochemical properties in acetonitrile. ${ }^{8}$ The fluorescence quantum yields $\left(\phi_{\mathrm{fl}}\right)$ were determined from the integration of the fluorescence, using an external standard of trans-stilbene in Ar-saturated $\mathrm{Bz}\left(\phi_{\mathrm{fl}}=\right.$ 0.048). ${ }^{11}$ The excitation energies of the lowest singlet excited state $\left(E_{\mathrm{S} 1}\right)$ were estimated from the peak wavelengths of the fluorescence spectra. The ground-state absorption peaks of $\mathbf{P 2 Q}$, $\mathbf{P 3 Q}$, and $\mathbf{P} 4^{\text {i }} \mathbf{Q}$ in $\mathrm{Bz}$ and acetonitrile were observed at 334$350 \mathrm{~nm}$ with a little influence of the solvent polarity (Supporting Information, Figure S1a, Table 1). The fluorescence peaks in Bz were observed at 373-394 nm (Figure 1a), while those in acetonitrile were at $405-414 \mathrm{~nm}$ with a large red shift due to the high polarity. With use of a setup consisting of a fluorescence spectrophotometer and a voltammograph with a PC interface, the ECL emission of P2Q (1.0 mM) was observed at $479 \mathrm{~nm}$ in acetonitrile with $0.05 \mathrm{M}$ tetrabutylammonium perchlorate (TBAP) as the supporting electrolyte. On the other hand, no ECL was observed in $\mathbf{P} \mathbf{3}$ and $\mathbf{P} 4 \mathbf{Q} \mathbf{Q}$ under the same conditions. ${ }^{8}$ The different emission behavior results are reasonably explained by the annihilation enthalpy change $\left(-\Delta H^{\circ}\right)$ for the charge recombination between $\mathbf{M}^{\bullet+}$ and $\mathbf{M}^{\bullet-}$, as expressed by eq $1,8,12$

$$
-\Delta H^{\circ}=E_{\mathrm{ox}}-E_{\text {red }}-0.16 \mathrm{eV}
$$

where $E_{\mathrm{ox}}$ and $E_{\text {red }}$ are the oxidation and reduction potentials of $\mathbf{M}$, respectively. The calculated $-\Delta H^{\circ}$ values for $\mathbf{P n Q}$ are listed in Table 1. For $\mathbf{P 2 Q},-\Delta H^{\circ}$ of the charge recombination between $\mathbf{P} \mathbf{Q}^{-+}$and $\mathbf{P} 2 \mathbf{Q}^{--}$is sufficiently larger than $E_{\mathrm{S} 1}$. Therefore, the charge recombination gives ${ }^{1} \mathbf{P} \mathbf{2} \mathbf{Q}^{*}$, leading to the efficient ECL.

For $\mathbf{P} 6 \mathbf{Q}_{\mathbf{a}-\mathbf{d}}$, the ground-state absorption peaks of $\mathbf{P} \mathbf{6} \mathbf{Q}_{\mathbf{a}-\mathbf{d}}$ in $\mathrm{Bz}$ and acetonitrile were observed at 313-372 $\mathrm{nm}$ with a little influence of the solvent polarity (Supporting Information, Figure $\mathrm{S} 1 \mathrm{~b}$, Table 1). The fluorescence peaks in $\mathrm{Bz}$ were observed at 369-434 nm (Figure 1b), while those in acetonitrile were at 364-536 nm with a large red shift due to the high polarity. It was shown that both absorption and fluorescence peaks shifted to the longer wavelength with increasing electron donating character of the substituent. In addition, the $E_{\mathrm{S} 1}$ values decrease and $\phi_{\mathrm{fl}}$ values increase with increasing electron donor character of the substituent. The ECL emissions of $\mathbf{P} 6 \mathbf{Q}_{\mathbf{a}-\mathbf{d}}(1.0 \mathrm{mM})$ were observed at $450-534 \mathrm{~nm}$ in acetonitrile. The $-\Delta H^{\circ}$ values of the charge recombination between $\mathbf{P} 6 \mathbf{Q}_{\mathbf{a}-\mathbf{d}^{+}}$and $\mathbf{P} 6 \mathbf{Q}_{\mathbf{a}-\mathbf{d}^{-}}{ }^{-}$ are not sufficient to populate their $E_{\mathrm{S} 1}$. Thus, the charge recombination may produce ${ }^{3} \mathbf{P} 6 \mathbf{Q}_{\mathbf{a}-\mathbf{d}}{ }^{*}$, which undergo the triplet-triplet annihilation to give ${ }^{1} \mathbf{P} 6 \mathbf{Q}_{\mathbf{a}-\mathbf{d}} *$. The compounds bearing weak donors $\left(\mathbf{P} 6 \mathbf{Q}_{\mathbf{a}-\mathbf{c}}\right)$ exhibit the ECL emission of the excimers because of the twisted angle between two planes of the donor and acceptor moieties. The ECL emission peak of $\mathbf{P 6 Q}_{\mathbf{d}}$ (534 nm) was blue shifted as compared with the solution photoluminescence peak $(536 \mathrm{~nm})$, suggesting the formation of an $H$-type excimer in which two quinolinyl moieties are stacked face-to-face with donor-bearing phenyl groups projecting perpendicularly away from each other. ${ }^{8}$ For $\mathbf{Q}$ and ${ }^{\mathrm{i}} \mathbf{Q}$, the fluorescence intensities were very weak.

Emission Spectra Observed during the Pulse Radiolysis of PnQ in Bz. The time-resolved emission spectra were recorded and they showed monotonic decay in the $40 \mathrm{~ns}$ time scale after the electron pulse during the pulse radiolysis of PnQ in Arsaturated $\mathrm{Bz}$ (Figures 2 and 3). The emission peaks of $\mathbf{P 4} \mathbf{Q}$ and $\mathbf{P} 6 \mathbf{Q}_{\mathbf{a}-\mathbf{d}}$ were observed at almost the same wavelengths where the fluorescence peaks were observed by the steady-state 


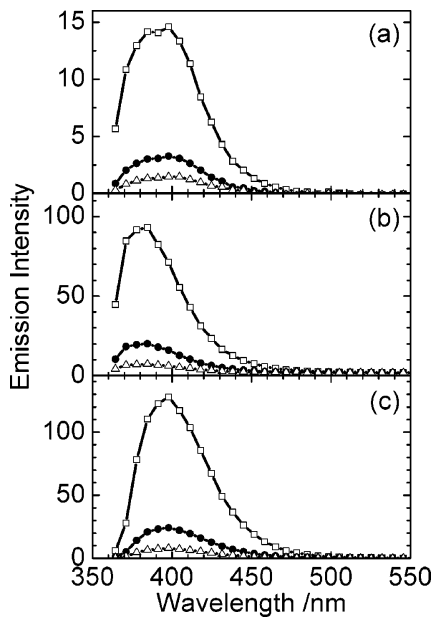

Figure 2. Time-resolved emission spectra recorded at time $t=10$ (open square), 20 (solid circle), and 30 (open triangle) ns after an electron pulse during the pulse radiolysis of P2Q (a), P3Q (b), and P4iQ (c) $(5.0 \mathrm{mM})$ in Ar-saturated Bz.

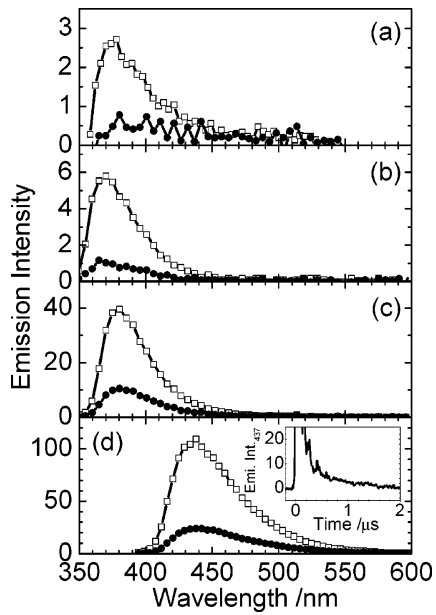

Figure 3. Emission spectra recorded at time $=10$ (open square) and 20 (solid circle) ns after an electron pulse during the pulse radiolysis of $\mathbf{P 6 Q} \mathbf{Q}_{\mathbf{a}}$ (a), $\mathbf{P 6} \mathbf{Q}_{\mathbf{b}}$ (b), $\mathbf{P 6} \mathbf{Q}_{\mathbf{c}}$ (c), and $\mathbf{P 6} \mathbf{Q}_{\mathbf{d}}$ (d) $(5.0 \mathrm{mM}$ ) in Ar-saturated Bz. Inset: Time profile of the emission of $\mathbf{P}^{6} \mathbf{Q}_{\mathbf{d}}$ at $437 \mathrm{~nm}$ in $\mathrm{Ar}-$ saturated Bz.

measurement. However, the emission peaks during the pulse radiolysis of P2Q and P3Q were observed at $10-20 \mathrm{~nm}$ longer wavelengths compared to the fluorescence peaks observed by the steady-state measurement. This is because of the selfabsorption as a result of the high concentrations ( $5 \mathrm{mM}$ ) of P2Q and P3Q in the pulse radiolysis experiment. It is confirmed that at high concentration, fluorescence peaks of $\mathbf{P 2 Q}$ and $\mathbf{P} 3 \mathbf{Q}$ in $\mathrm{Bz}$ were also observed at a longer wavelength. For $\mathbf{Q}$ and ${ }^{\mathbf{i}} \mathbf{Q}$, no emission was observed during the pulse radiolysis. Contrary to PnQs, very weak emissions of PAs were observed during the pulse radiolysis. The emission spectral data of $\mathbf{P n Q}, \mathbf{Q},{ }^{\mathrm{i}} \mathbf{Q}$, and PAs obtained during the pulse radiolysis are summarized in Table 2, together with the ECL emission data. The relative emission intensity was determined from the integration of the emission spectrum observed at $10 \mathrm{~ns}$ after an electron pulse during the pulse radiolysis.

It should be noted that all PnQ show emissions with peaks at $370-437 \mathrm{~nm}$ in the $40 \mathrm{~ns}$ time scale after the electron pulse during the pulse radiolysis. The donor moieties of $\mathbf{P n Q}$ are toluene and anisole auxochrome having the fluorescence peaks at $\mathbf{2 8 0}-300 \mathrm{~nm}$, while the acceptor moiety of $\mathbf{P n Q}$ is $\mathbf{Q}$ or ${ }^{\mathrm{i}} \mathbf{Q}$ having the fluorescence peaks at $350-500 \mathrm{~nm}$. Therefore, the emission from the pulse radiolysis originates from the acceptor
TABLE 2: Emission Peak $\left(\lambda_{\text {Em }}^{\text {Em }}\right)$ and Relative Emission Intensity of PnQ, $Q$, ${ }^{\mathrm{Q}} \mathrm{Q}$, and $\mathrm{PAs}$ Observed at $10 \mathrm{~ns}$ after an Electron Pulse during the Pulse Radiolysis, Together with the ECL Emission Peak and Intensity

\begin{tabular}{|c|c|c|c|c|}
\hline \multirow[b]{2}{*}{ compd } & \multicolumn{2}{|c|}{ in $\mathrm{Bz}$} & \multicolumn{2}{|c|}{ in $\mathrm{CH}_{3} \mathrm{CN}$} \\
\hline & $\lambda_{\max }^{\mathrm{Em}} / \mathrm{nm}$ & $\begin{array}{c}\text { rel emission } \\
\text { intensity }^{a}(\%)\end{array}$ & $\lambda_{\max }^{\mathrm{ECL}} / \mathrm{nm}$ & $\begin{array}{c}\text { ECL } \\
\text { intensity }^{c}(\%)\end{array}$ \\
\hline P2Q & 395 & 16.9 & 479 & $37.8^{d}$ \\
\hline P3Q & 383 & 100 & $b$ & \\
\hline P4iQ & 395 & 144 & $b$ & \\
\hline$P_{6} Q_{a}$ & 370 & 2.97 & 460 & 6.0 \\
\hline $\mathbf{P 6 Q}_{\mathrm{b}}$ & 370 & 5.74 & 450 & 1.5 \\
\hline $\mathbf{P 6 Q}_{\mathrm{c}}$ & 379 & 38.5 & 475 & 1.2 \\
\hline $\mathbf{P 6 Q}_{\mathrm{d}}$ & 437 & 143 & 534 & 0.5 \\
\hline $\mathbf{Q}$ & $b$ & 0 & $b$ & \\
\hline $\mathbf{Q}$ & $b$ & 0 & $b$ & \\
\hline $\mathbf{P A}$ & 378 & 0.85 & $b$ & \\
\hline DPA & 338,467 & 2.06 & $520^{e}$ & 7.0 \\
\hline $\mathbf{D P A}_{b}$ & 422,577 & 0.41 & $b$ & \\
\hline
\end{tabular}

${ }^{a}$ Relative to the intensity for $\mathbf{P 3 Q}=100 \%$. Emission intensity was obtained by integration of emission observed at all wavelengths at 10 ns after an electron pulse irradiation. ${ }^{b}$ No or very weak emission was observed. ${ }^{c}$ Relative to the intensity for tris(2,2-bipyridyl)ruthenium(II) oxalate complex $=100 \% .{ }^{d}$ Reference $8 \mathrm{~b} .{ }^{e}$ Observation of the ECL at this wavelength for DPA is quite unusual.

moiety. According to the $-\Delta H^{\circ}$ values, no emission should be observed during the pulse radiolysis of $\mathbf{P} \mathbf{4}^{\mathbf{i}} \mathbf{Q}$ in $\mathrm{Bz}$, because ${ }^{1} \mathbf{P} 4 \mathbf{Q} \mathbf{Q}^{*}$ cannot be generated from the charge recombination between $\mathbf{P} 4^{\mathbf{i}} \mathbf{Q}^{\bullet+}$ and $\mathbf{P} \mathbf{4}^{\mathbf{i}} \mathbf{Q}^{\bullet-}$. However, $\mathbf{P} \mathbf{4}^{\mathbf{i}} \mathbf{Q}$ showed the strongest fluorescence during the pulse radiolysis of $\mathbf{P n Q}$. Therefore, the occurrence of the efficient emission during the pulse radiolysis of $\mathbf{P n Q}$ cannot be explained by the $-\Delta H^{\circ}$ value for the charge recombination between $\mathbf{P n} \mathbf{Q}^{\cdot+}$ and $\mathbf{P n} \mathbf{Q}^{\bullet-}$.

The emission intensities of $\mathbf{P} \mathbf{6} \mathbf{Q}_{\mathbf{a}-\mathbf{d}}$ observed during the pulse radiolysis were much higher than those of $\mathbf{Q}$ and the corresponding PAs. For example, the emission intensity of $\mathbf{P} 6 \mathbf{Q}_{\mathbf{b}}$ was 14 times higher than that of $\mathbf{D P A} \mathbf{A}_{\mathbf{b}}$. This result indicates that the strong emission is due to the strong electronic coupling between the donor and acceptor through conjugation of $\mathbf{P} \mathbf{6} \mathbf{Q}_{\mathbf{a}-\mathbf{d}}$. In addition, for $\mathbf{P} \mathbf{6} \mathbf{Q}_{\mathbf{d}}$ the long-lifetime emission was observed during the pulse radiolysis (Figure $3 \mathrm{~d}$, inset). It should be noted that the emission intensity, observed during the pulse radiolysis of $\mathbf{P} 6 \mathbf{Q}_{\mathbf{a}-\mathbf{d}}$, increased with increasing electron donor character of the substituents. This tendency is different from that observed for the ECL of $\mathbf{P} 6 \mathbf{Q}_{\mathbf{a}-\mathbf{d}}$.

Transient Absorption Spectra Observed during the Pulse Radiolysis of PnQ. To generate both $\mathbf{P n Q} \mathbf{Q}^{-+}$and $\mathbf{P n} \mathbf{Q}^{--}$and to observe the annihilation of $\mathbf{P n} \mathbf{Q}^{\bullet+}$ and $\mathbf{P n} \mathbf{Q}^{--}$through the charge recombination by energetic collision of the charged species, Bz was used as solvent for the pulse radiolysis of $\mathbf{M}$. Figure 4 shows the transient absorption spectra observed during the pulse radiolysis of $\mathbf{P} \mathbf{2}, \mathbf{P} 3 \mathbf{Q}$, and $\mathbf{P} \mathbf{4}^{\mathbf{i}} \mathbf{Q}$ in Ar-saturated $\mathrm{Bz}$. The transient absorption spectra of $\mathrm{Bz}^{\cdot+}$ and ${ }^{3} \mathrm{Bz}^{*}$ have been reported to have a peak at 400 and $235 \mathrm{~nm}$ with very small molar absorption coefficients, $\epsilon_{400}=1.2 \times 10^{3} \mathrm{dm}^{3} \mathrm{~mol}^{-1} \mathrm{~cm}^{-1}$ and $\epsilon_{235}=1.1 \times 10^{4} \mathrm{dm}^{3} \mathrm{~mol}^{-1} \mathrm{~cm}^{-1}$, respectively. ${ }^{14}$ The transient absorption spectrum of $\mathrm{e}^{-} \mathrm{S}$ in $\mathrm{Bz}$ has not been reported. It is assumed that $\mathrm{e}^{-} \mathrm{S}$ is rapidly trapped by $\mathbf{P n Q}$ or undergoes the fast geminate charge recombination. Therefore, these species cannot be observed. The transient absorption spectra observed at $t=50 \mathrm{~ns}$ after an electron pulse in Figure 4 can be assigned to $\mathbf{P n} \mathbf{Q}^{\bullet+}, \mathbf{P n Q}^{\bullet-}$, and/or ${ }^{3} \mathbf{P n} \mathbf{Q}^{*}$.

To confirm the transient absorption spectra of $\mathbf{P n} \mathbf{Q}^{\bullet+}$ and $\mathbf{P n Q}^{-}$, the pulse radiolysis of PnQ in DCE and DMF was examined. It is established that $\mathbf{M}^{\bullet+}$ is generated during the pulse 


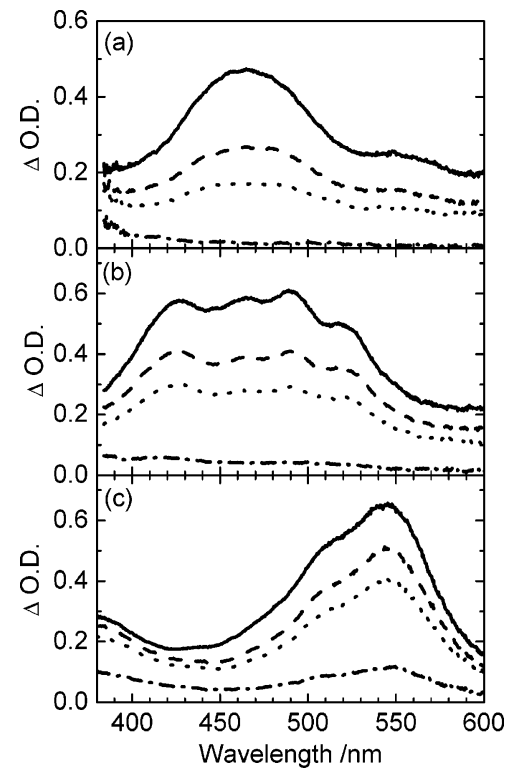

Figure 4. Transient absorption spectra observed at time $t=50$ (solid line) ns, 500 (broken line) ns, 1 (dotted line) $\mu \mathrm{s}$, and 5 (broken and dotted line) $\mu$ s after an electron pulse $(8 \mathrm{~ns})$ during the pulse radiolysis of P2Q (a), P3Q (b), and P4iQ (c) $(5.0 \mathrm{mM})$ in Ar-saturated Bz.

SCHEME 4: Pulse Radiolysis of $M$ in DCE

$$
\begin{aligned}
& \mathrm{DCE} \rightarrow \mathrm{DCE}^{\bullet+}+\mathrm{e}_{\mathrm{s}}^{-} \\
& \mathrm{DCE}^{\bullet+}+\mathbf{M} \rightarrow \mathbf{M}^{\bullet+}+\mathrm{DCE} \\
& \mathrm{e}_{\mathrm{s}}^{-}+\mathrm{DCE} \rightarrow \mathrm{ClCH}_{2} \mathrm{CH}_{2}{ }^{\bullet}+\mathrm{Cl}^{-}
\end{aligned}
$$

\section{SCHEME 5: Pulse Radiolysis of $M$ in DMF}

$$
\begin{aligned}
& \mathrm{DMF} \rightarrow \mathrm{DMF}^{\bullet+}+\mathrm{e}_{\mathrm{s}}^{-} \\
& \mathrm{e}_{\mathrm{s}}^{-}+\mathbf{M} \rightarrow \mathbf{M}^{\bullet-} \\
& \mathrm{DMF}^{\bullet+}+\mathrm{DMF} \rightarrow \operatorname{DMF}\left(-\mathrm{H}^{+}\right)^{\bullet}+\mathrm{DMF}\left(+\mathrm{H}^{+}\right)^{+}
\end{aligned}
$$

radiolysis of $\mathbf{M}$ in DCE, ${ }^{2}$ while $\mathbf{M}^{\boldsymbol{0}^{-}}$is in $\mathrm{DMF}^{3}$ as shown in Schemes 4 and 5, respectively.

Figure 5a shows the transient absorption spectrum observed during the pulse radiolysis of $\mathbf{P 2 Q}$ in Ar-saturated DCE. Although a peak at $465 \mathrm{~nm}$ disappeared, that around $430 \mathrm{~nm}$ remained even at $>5 \mu \mathrm{s}$. Therefore, the absorption around 430$450 \mathrm{~nm}$ is assumed to be a stable product, while the peak observed at $465 \mathrm{~nm}\left(\tau_{1 / 2}=0.25 \mu \mathrm{s}\right)$ is assigned to $\mathbf{P 2} \mathbf{Q}^{\bullet+}$. It seems that $\mathrm{Cl}^{-}$reacts with $\mathbf{P 2} \mathbf{Q}^{+}$to give the product having an absorption peak around $430-450 \mathrm{~nm}$. Figure $5 \mathrm{~b}$ shows the transient absorption spectrum observed during the pulse radiolysis of P2Q in Ar-saturated DMF. The peak observed at 449 $\mathrm{nm}\left(\tau_{1 / 2}=0.67 \mu \mathrm{s}\right)$ is assigned to $\mathbf{P} 2 \mathbf{Q}^{--}$. Similarity, the transient absorption spectra assigned to $\mathbf{P n} \mathbf{Q}^{\cdot+}$ and $\mathbf{P n} \mathbf{Q}^{--}$were observed during the pulse radiolysis of other PnQ in DCE and DMF, respectively (Supporting Information, Figures S2 and S3). These results are summarized in Table 3 . Since the transient absorption spectra of $\mathbf{P n Q} \mathbf{Q}^{+}$and $\mathbf{P n} \mathbf{Q}^{--}$have peaks around 430-500 nm in DCE and DMF, respectively, $\mathbf{P n} \mathbf{Q}^{++}$and $\mathbf{P n} \mathbf{Q}^{--}$ seem to be involved in the transient absorption spectra, observed during the pulse radiolysis of PnQ in Bz. However, it is not clear whether ${ }^{1} \mathbf{P n Q} \mathbf{Q}^{*}$ is involved in the transient absorption spectra or not.

Emission and Absorption Spectra Observed during the Laser Flash Photolysis of PnQ in Bz. To assign the transient species in detail, the transient absorption spectra of $\mathbf{P n Q}$ in $\mathrm{Bz}$

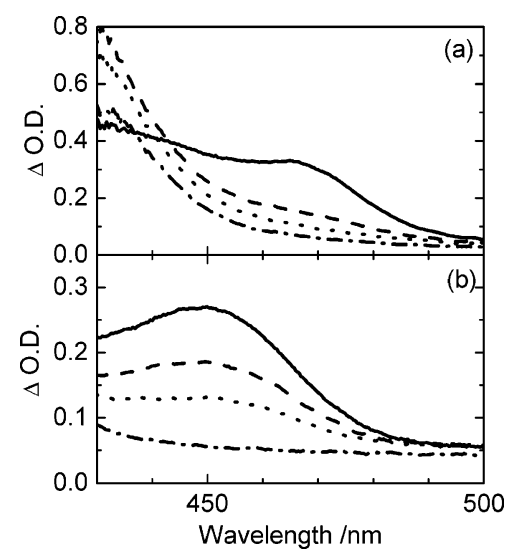

Figure 5. Transient absorption spectra observed at time $t=50$ (solid line) ns, 500 (broken line) ns, 1 (dotted line) $\mu$ s, and 5 (broken and

\begin{tabular}{|c|c|c|c|c|c|c|}
\hline \multirow[b]{2}{*}{ compd } & \multicolumn{2}{|c|}{ in $\mathrm{Bz}$} & \multicolumn{2}{|c|}{ in DCE } & \multicolumn{2}{|c|}{ in DMF } \\
\hline & $\lambda_{\max } / \mathrm{nm}$ & $\tau_{1 / 2} / \mu \mathrm{s}$ & $\lambda_{\max } / \mathrm{nm}$ & $\tau_{1 / 2} / \mu \mathrm{s}$ & $\lambda_{\max } / \mathrm{nm}$ & $\tau_{1 / 2} / \mu \mathrm{s}$ \\
\hline P2Q & 465 & 53 & 465 & 0.25 & 449 & 0.67 \\
\hline P3Q & $\begin{array}{l}427,465, \\
\quad 489,517\end{array}$ & 0.70 & 5,483 & 0.35 & 456 & .70 \\
\hline P4iQ & 545 & 1.1 & 490,528 & 0.3 & 467,505 & 0.40 \\
\hline$P 6 Q_{a}$ & 427,447 & 0.7 & 452,471 & 0.2 & 359,471 & 2.2 \\
\hline $\mathbf{P 6 Q}_{\mathrm{b}}$ & 433,453 & 0.68 & 456,478 & 0.15 & 469 & 0.51 \\
\hline $\mathbf{P 6 Q}_{\mathrm{c}}$ & 473 & 0.68 & 453,479 & 0.30 & 452 & 0.36 \\
\hline$P_{6} Q_{d}$ & 603,658 & 0.60 & $600^{a}$ & 4.3 & 470 & 0.30 \\
\hline Q & 400 & 1.3 & 402 & & 398 & 0.45 \\
\hline${ }^{\mathrm{Q}} \mathbf{Q}$ & 420,540 & 1.3 & 418,546 & 0.019 & 416 & 0.34 \\
\hline
\end{tabular}
dotted line) $\mu \mathrm{s}$ after an electron pulse (8 ns) during the pulse radiolysis of P2Q (5.0 mM) in Ar-saturated DCE (a) and DMF (b).

TABLE 3: Transient Absorption Peaks $\left(\lambda_{\max }\right)$ and Half Lifetimes $\left(\tau_{1 / 2}\right)$ Obtained during the Pulse Radiolysis of PnQ in Ar-Saturated Bz, DCE, and DMF

${ }^{a}$ A broad transient absorption band was observed.

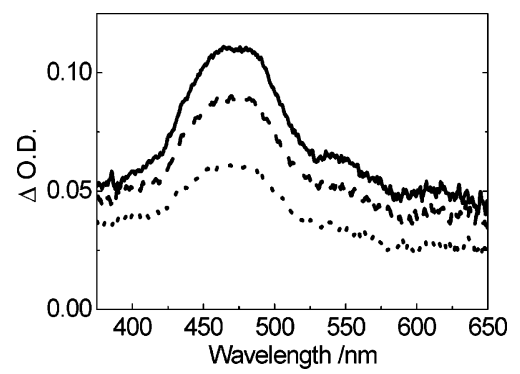

Figure 6. Time-resolved transient absorption spectrum at time $t=3$ (solid line), 5 (broken line), and 10 (dotted line) $\mu$ s after a laser pulse during the laser flash photolysis of $\mathbf{P 2 Q}(20 \mu \mathrm{M})$ in Ar-saturated Bz.

were measured during the 355-nm 5-ns laser flash photolysis (Supporting Information, Figure S4). A transient absorption spectrum with a peak at $470 \mathrm{~nm}$ was observed during the laser flash photolysis of $\mathbf{P 2 Q}$ in Ar-saturated Bz (Figure 6). During the laser flash photolysis under the one-photon excitation condition, the most probable long-lived transient is the ${ }^{3} \mathbf{P} 2 \mathbf{Q}^{*}$, which is formed from intersystem crossing of ${ }^{1} \mathbf{P} 2 \mathbf{Q}^{*}$. Therefore, the transient absorption spectrum, observed during the pulse radiolysis of $\mathbf{P 2 Q}$ in $\mathrm{Ar}$-saturated $\mathrm{Bz}$, is also assigned to mainly ${ }^{3} \mathbf{P} 2 \mathbf{Q}^{*}$. It is assumed that $\mathbf{P} 2 \mathbf{Q}^{-+}$and $\mathbf{P} 2 \mathbf{Q}^{--}$immediately recombine to give ${ }^{1} \mathbf{P} 2 \mathbf{Q}^{*}$ and ${ }^{3} \mathbf{P} 2 \mathbf{Q}^{*}$, that ${ }^{1} \mathbf{P} 2 \mathbf{Q}^{*}$ emits light within a pulse duration, and that ${ }^{3} \mathbf{P} 2 \mathbf{Q}^{*}$ remains in the microsecond time scale.

Emission spectra of $\mathbf{P n Q}$ were also measured during the laser flash photolysis. Emissions with extremely long lifetimes (microsecond time scale) were observed for P2Q (Figure 7), $\mathbf{P 3 Q}, \mathbf{P 4} \mathbf{Q}$, and $\mathbf{P 6} \mathbf{Q}_{\mathbf{d}}$ (Supporting Information, Figure S5). 


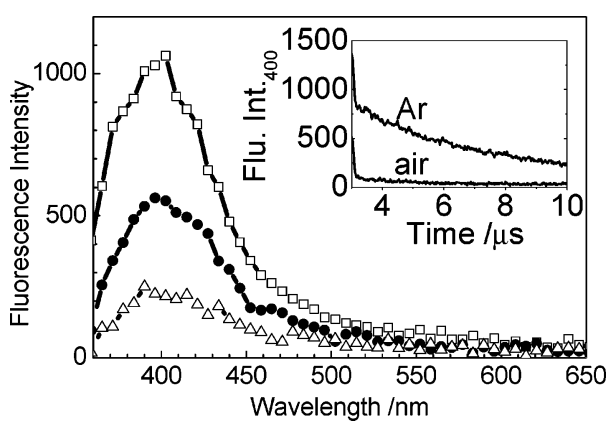

Figure 7. Time-resolved emission spectrum at time $t=3$ (open square), 5 (solid circle), and 10 (open triangle) $\mu$ s after a laser pulse during the laser flash photolysis of P2Q $(20 \mu \mathrm{M})$ in Ar-saturated Bz. Inset: Time profile of emission at $400 \mathrm{~nm}$ with Ar- and air-saturated $\mathrm{Bz}$.

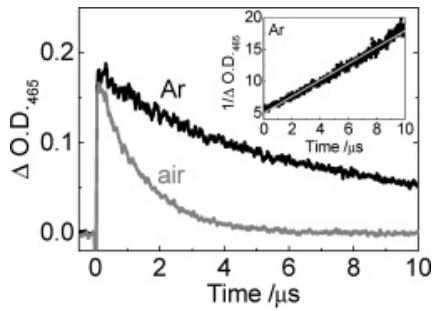

Figure 8. Time profiles of transient absorption at $465 \mathrm{~nm}$ during the laser flash photolysis of P2Q $(20 \mu \mathrm{M})$ in Ar- and air-saturated Bz. Inset: Time profile of $\Delta \mathrm{OD}_{465}{ }^{-1}$ in Ar-saturated Bz.

Because the emission intensities of these compounds were significantly high, we did not measure the emission in the time scale of $0-3 \mu \mathrm{s}$ after the $355-\mathrm{nm} 5$-ns laser pulse using the streak camera or the silicon detector. Under air-saturated conditions, the long-lived emissions were completely quenched by oxygen through the triplet energy transfer quenching mechanism (Figure 7, inset, and Figure 8), suggesting that the emission originated from ${ }^{3} \mathbf{P} 2 \mathbf{Q}^{*}$. The time profile of $\Delta \mathrm{OD}_{465}{ }^{-1}$ shows the decay of ${ }^{3} \mathbf{P} 2 \mathbf{Q}^{*}$ obeys second-order kinetics (Figure 8 , inset). These results suggest that the long lifetime emission corresponds to the "P-type" delayed fluorescence from ${ }^{1} \mathbf{P} 2 \mathbf{Q}^{*}$ generated from the triplet-triplet annihilation. ${ }^{15}$

For $\mathbf{P 6}_{\mathbf{a}-\mathbf{c}}$, the peaks assigned to the excimer emission were observed at $t=500 \mathrm{~ns}$ at around $500 \mathrm{~nm}$ (Supporting Information, Figure S5). This emission is assumed to be derived from the excimer generated from the triplet-triplet annihilation. For $\mathbf{P 6} \mathbf{Q}_{\mathbf{d}}$, the long-lifetime emission was observed during the laser flash photolysis. This emission is also assigned to be the "P-type" delayed fluorescence derived from the triplet-triplet annihilation. The long-lifetime emission was also observed during the pulse radiolysis of $\mathbf{P} \mathbf{6} \mathbf{Q}_{\mathbf{d}}$ and can be assigned to the "P-type" delayed fluorescence (Figure 3d, inset). In the electrochemical reactions of $\mathbf{P} \mathbf{6} \mathbf{Q}_{\mathbf{a}-\mathbf{d}}$, it is assumed that the emission pathway occurs through the triplet-triplet annihilation. No ECL emission was observed for $\mathbf{P 3 Q}$ and $\mathbf{P} \mathbf{4}^{\mathbf{i}} \mathbf{Q}$ in which the donor arylethynyl groups are in a meta-relationship on the ring nitrogen. ${ }^{8}$ Therefore, it is expected that the efficient emission from $\mathbf{P 3 Q}$ and $\mathbf{P 4} \mathbf{Q}_{\mathbf{Q}}$ was generated mainly from the charge recombination between $\mathbf{P n} \mathbf{Q}^{\bullet+}$ and $\mathbf{P n} \mathbf{Q}^{\bullet-}$ during the pulse radiolysis. For $\mathbf{P} \mathbf{6} \mathbf{Q}_{\mathbf{a}-\mathbf{d}}$, it is also assumed that the efficient monomer emission was derived from ${ }^{1} \mathbf{P} 6 \mathbf{Q}_{\mathbf{a}-\mathbf{d}}{ }^{*}$ generated from the charge recombination and/or triplet-triplet annihilation during the pulse radiolysis in Bz. It is suggested that the efficient emission from ${ }^{1} \mathbf{P} 6 \mathbf{Q}_{\mathbf{a}-\mathbf{d}} *$ during the pulse radiolysis of $\mathbf{P} \mathbf{6} \mathbf{Q}_{\mathbf{a}-\mathbf{d}}$ is also caused mainly from the charge recombination, because the higher emission values were observed for $\mathbf{P} 3 \mathbf{Q}$ and $\mathbf{P} 4{ }^{\mathbf{Q}} \mathbf{Q}$ during the pulse radiolysis in Bz.
TABLE 4: Oxidation Potentials $\left(E_{0 x}\right)$ of Donor Moiety, Reduction Potentials $\left(E_{\text {red }}\right)$ of Acceptor Moiety, Estimated Annihilation Enthalpy Values $\left(-\Delta H^{\circ}\right)$ for the Charge Recombination between $\mathrm{P}^{-+}$and $\mathrm{P}^{\bullet} \mathrm{Q}^{\bullet-}$ with Localized Charges on the Donor and Acceptor Moieties, and $E_{\mathrm{S} 1}$ of PnQ

\begin{tabular}{llllllc}
\hline & \multicolumn{2}{c}{ in $\mathrm{CH}_{3} \mathrm{CN}$} & \multicolumn{2}{c}{ in $\mathrm{CH}_{3} \mathrm{CN}$} & in $\mathrm{Bz}$ \\
\hline compd & \multicolumn{1}{c}{ donor } & $E_{\text {ox }}{ }^{a} / \mathrm{V}$ & acceptor & $E_{\text {red }}{ }^{a} / \mathrm{V}$ & $-\Delta H^{\prime \circ} / \mathrm{eV}$ & $E_{\mathrm{S} 1} / \mathrm{eV}$ \\
\hline $\mathbf{P 2 Q}$ & anisole & $1.35^{b}$ & $\mathbf{Q}$ & $-2.40^{f}$ & 3.59 & 3.31 \\
$\mathbf{P 3 Q}$ & anisole & 1.35 & $\mathbf{Q}$ & -2.40 & 3.59 & 3.32 \\
$\mathbf{P 4} \mathbf{Q}$ & anisole & 1.35 & ${ }^{i} \mathbf{Q}$ & $-2.51^{f}$ & 3.70 & 3.15 \\
$\mathbf{P 6 Q}$ & benzene & $2.08^{c}$ & $\mathbf{Q}$ & -2.40 & 4.32 & 3.36 \\
$\mathbf{P 6 Q}$ & toluene & $1.98^{c, d}$ & $\mathbf{Q}$ & -2.40 & 4.22 & 3.34 \\
$\mathbf{P 6 Q}$ & anisole & $1.35^{d}$ & $\mathbf{Q}$ & -2.40 & 3.59 & 3.28 \\
$\mathbf{P 6 Q}$ & $N, N$-diethyl- & $0.47^{e}$ & $\mathbf{Q}$ & -2.40 & 2.71 & 2.86 \\
& aniline & & & & &
\end{tabular}

${ }^{a}$ Redox potentials were estimated vs $\mathrm{Ag} / \mathrm{Ag}^{+} .{ }^{b}$ Reference $16 \mathrm{a}$ ${ }^{c}$ Reference 16b. ${ }^{d}$ Reference $16 \mathrm{c} .{ }^{e}$ Reference $16 \mathrm{~d} .{ }^{f}$ Reference 13a.

SCHEME 6: Mechanism for the Emission during the Pulse Radilolysis of PnQ $(A-D)$ in $B z$

$$
\begin{aligned}
& \mathrm{e}_{\mathrm{s}}^{-}+\mathrm{A}-\mathrm{D} \rightarrow \mathrm{A}^{\bullet-}-\mathrm{D} \\
& \mathrm{Bz}^{\bullet+}+\mathrm{A}-\mathrm{D} \rightarrow \mathrm{Bz}+\mathrm{A}-\mathrm{D}^{\bullet+} \\
& \mathrm{A}^{\bullet-}-\mathrm{D}+\mathrm{A}-\mathrm{D}^{\bullet+} \rightarrow{ }^{1}(\mathrm{~A}-\mathrm{D})^{*}+\mathrm{A}-\mathrm{D} \\
& { }^{1}(\mathrm{~A}-\mathrm{D})^{*} \rightarrow \mathrm{A}-\mathrm{D}+h v_{\mathrm{fl}}
\end{aligned}
$$

Emission Mechanism. Because the efficient emission of PnQ seems to result from the donor-acceptor structure, the characteristic emission mechanism for the donor-acceptor molecules should be assumed. It is suggested that negative charge is localized on the electron acceptor moiety in the radical anion $\left(\mathrm{A}^{\bullet-}-\mathrm{D}\right)$. On the other hand, positive charge is localized on the electron donor moiety in the radical cation $\left(\mathrm{A}-\mathrm{D}^{\bullet+}\right)$. This scheme reasonably explains the emission mechanism during the pulse radiolysis of $\mathbf{P n Q}$. For $\mathbf{P} 2 \mathbf{Q}$, the $-\Delta H^{\circ}$ value of the donor moiety (corresponding to anisole) is $1.35 \mathrm{~V}$, while that of the acceptor moiety (corresponding to $\mathbf{Q}$ ) is $-2.40 \mathrm{~V}$. Therefore, the $-\Delta H^{\circ}$ value for the charge recombination between $\mathbf{P} 2 \mathbf{Q}^{\cdot+}$ and $\mathbf{P} 2 \mathbf{Q}^{--}$with localized charges can be estimated to be 3.59 $\mathrm{eV}$, which is sufficiently larger than the $E_{\mathrm{S} 1}$ value. Similarly, the $-\Delta H^{\prime \circ}$ values for $\mathbf{P} 3 \mathbf{Q}, \mathbf{P} 4 \mathbf{Q} \mathbf{Q}$, and $\mathbf{P} 6 \mathbf{Q}_{\mathbf{a}-\mathbf{c}}$ are larger than the $E_{\mathrm{S} 1}$ values of ${ }^{1} \mathbf{P n} \mathbf{Q}^{*}$ (Table 4$)$. Therefore, the charge recombination between $\mathrm{A}^{\bullet-}-\mathrm{D}$ and $\mathrm{A}-\mathrm{D}^{\bullet+}$ occurs to give ${ }^{1} \mathbf{P n} \mathbf{Q}^{*}$ during the pulse radiolysis of PnQ in Bz (Scheme 6). The emission intensity seems to depend on the $\phi_{\mathrm{fl}}$ values of $\mathbf{P n Q}$.

However, the $-\Delta H^{\prime \circ}$ value of $\mathbf{P} 6 \mathbf{Q}_{\mathbf{d}}$ is not sufficient to populate ${ }^{1} \mathbf{P n Q} \mathbf{Q}^{*}$. Therefore, it is suggested that the emission mechanisum for $\mathbf{P} 6 \mathbf{Q}_{\mathbf{d}}$ is quite different from that for other $\mathbf{P n Q}$. For $\mathbf{P 6} \mathbf{Q}_{\mathbf{d}}$ bearing a strong donor $\left(\mathrm{NEt}_{2}\right)$, the emissive singlet intramolecular charge transfer (ICT) state $\left({ }^{1}\left(\mathrm{~A}^{\bullet-}-\mathrm{D}^{\bullet+}\right)^{*}\right)$ with the larger twisted angle is assumed. ${ }^{8,17}$ The ICT state for $\mathbf{P} 6 \mathbf{Q}_{\mathbf{d}}$ is also expected by the slightly longer $\tau_{\mathrm{f}}$ than those of other PnQ (Table 1).

The emission mechanism during the pulse radiolysis of $\mathbf{P n Q}$ can be divided into two categories. For P2Q, P3Q, P4 $\mathbf{Q}$, and $\mathbf{P 6 Q}_{\mathbf{a}-\mathbf{c}}$ with no or weak electron donating substituents and no or small twist angle between the donor and acceptor planes, it is less favorable to populate the emissive singlet ICT state. For $\mathbf{P} 6 \mathbf{Q}_{\mathbf{d}}$ with a strong electron donating substituent and large twist angle between the donor and acceptor planes, the emission mechanism is quite different from that for other PnQ. It is suggested that the radical cation and anion collide neck-to-neck 
SCHEME 7: Proposed Structure for the Formation of ICT State $\left({ }^{1}\left(A^{\bullet-}-D^{\bullet+}\right)^{*}\right)$ during the Pulse Radiolysis of P6Q $Q_{d}$ in Bz

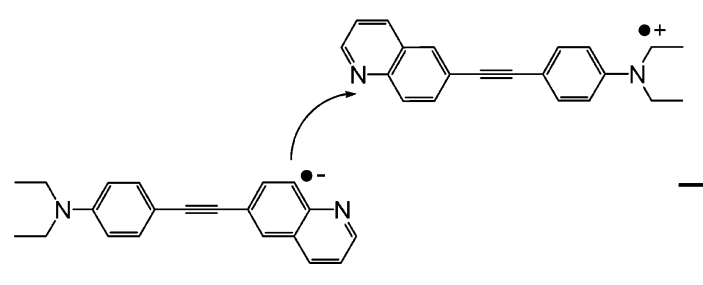

to produce the singlet ICT state $\left({ }^{1}\left(\mathrm{~A}^{\bullet-}-\mathrm{D}^{\bullet+}\right)^{*}\right)($ Scheme 7$) .^{8} \mathrm{It}$ is assumed that the $-\Delta H^{\circ}$ value of $\mathbf{P} 6 \mathbf{Q}_{\mathbf{d}}$ is sufficient to populate the singlet ICT state $\left({ }^{1}\left(\mathrm{~A}^{\bullet-}-\mathrm{D}^{\bullet+}\right)^{*}\right)$, although it is deficient to populate the singlet state $\left({ }^{1}(\mathrm{~A}-\mathrm{D})^{*}\right)$, therefore the efficient emission derived from the directly formed ICT state can be observed during the pulse radiolysis. The emission is possibly derived from ${ }^{1} \mathbf{P} \mathbf{6} \mathbf{Q}_{\mathbf{d}}{ }^{*}$, which is generated from the triplet-triplet annihilation. However, this cannot explain the higher emission intensity obtained during the pulse radiolysis (Table 2), because the emission pathway through the triplettriplet annihilation is assumed to be inefficient.

In the electroluminescence experiment, the emission peaks of P2Q and $\mathbf{P 6} \mathbf{Q}_{\mathbf{a}-\mathbf{d}}$ in acetonitrile were observed at $450-534$ nm. For P2Q, it has reported that the P2Q excimer $\left({ }^{1}(\mathbf{P} 2 \mathbf{Q})_{2} *\right)$ is the emissive species in the electrochemical reaction in acetonitrile (Scheme 2). ${ }^{8}$ On the other hand, the emission peaks were observed at $370-437 \mathrm{~nm}$ during the pulse radiolysis of PnQ in Bz, almost same as those observed by the steady-state measurement at concentrations of $20 \mu \mathrm{M}$ to $5 \mathrm{mM}$. Therefore, the emissive species is assigned to ${ }^{1} \mathbf{P n} \mathbf{Q}^{*}$ during the pulse radiolysis of $\mathbf{P n Q}$ in $\mathrm{Bz}$. Because $\mathbf{P n Q}^{\bullet+}$ and $\mathbf{P n Q}^{\bullet-}$ are generated as the initial species during both pulse radiolysis and electrochemical reaction, the difference of the emissive species corresponds to other experimental conditions such as the solvent and concentrations of $\mathbf{P n Q}, \mathbf{P n} \mathbf{Q}^{\bullet+}$, and $\mathbf{P n} \mathbf{Q}^{\bullet-}$. It is suggested that the formation of ${ }^{1}(\mathbf{P n Q})_{2} *$ was enhanced by the stabilization in acetonitrile as a polar solvent, and with higher concentrations of $\mathbf{P n Q}, \mathbf{P n} \mathbf{Q}^{\bullet+}$, and $\mathbf{P n} \mathbf{Q}^{\bullet-}$ during the electrochemical reaction.

\section{Conclusions}

Various donor-acceptor quinolines PnQ with an ethynyl linkage, which are known as the efficient ECL molecules by the electrochemical reaction, showed the efficient emission as well during the pulse radiolysis in Bz. The emission is suggested to originate from ${ }^{1} \mathbf{P n} \mathbf{Q}^{*}$ generated from the charge recombination between $\mathbf{P n} \mathbf{Q}^{\bullet+}$ and $\mathbf{P n} \mathbf{Q}^{\boldsymbol{}^{-}}$which yield from the initial radiolytic reaction in $\mathrm{Bz}$. The emission intensities of $\mathbf{P n Q}$, observed at $10 \mathrm{~ns}$ after an electron pulse, were 1.5-70 times higher than that of DPA during the pulse radiolysis. These results indicate that the efficient emission is due to the strong electronic coupling between the donor and acceptor through conjugation of PnQ. It is suggested that the positive and negative charges in $\mathbf{P n Q}^{\mathbf{}}{ }^{+}$and $\mathbf{P n} \mathbf{Q}^{--}$are localized on the donor (substituted phenyl groups) and acceptor $(\mathbf{Q})$ moieties, respectively. For P2Q, P3Q, P4i $\mathbf{Q}$, and $\mathbf{P 6} \mathbf{Q}_{\mathbf{a}-\mathbf{c}}$, the estimated $-\Delta H^{\circ}$ values $(3.6-4.3 \mathrm{eV})$ for the charge recombination between $\mathbf{P n Q}^{\bullet+}$ and $\mathbf{P n Q}^{--}$with localized charges are estimated from $E_{\text {ox }}$ of substituted phenyl groups and $E_{\text {red }}$ of $\mathbf{Q}$ to be sufficiently larger than $E_{\mathrm{S} 1}$ of PnQ $(2.8-3.3 \mathrm{eV})$. For $\mathbf{P} 6 \mathbf{Q}_{\mathbf{d}}$, the $-\Delta H^{\prime \circ}$ value $(2.71 \mathrm{eV})$ is not sufficiently larger than $E_{\mathrm{S} 1}$ of $\mathbf{P} 6 \mathbf{Q}_{\mathbf{d}}(2.86$ $\mathrm{eV}$ ), suggesting the formation of the singlet ICT state, which emits light generated from the charge recombination due to the strong electron donating substituent. Therefore, it is confirmed that $\mathbf{P n} \mathbf{Q}^{\bullet+}$ and $\mathbf{P n} \mathbf{Q}^{--}$are generated during the pulse radiolysis of $\mathbf{P n Q}$ in $\mathrm{Bz}$ and that the emission is responsible for ${ }^{1} \mathbf{P n} \mathbf{Q}^{*}$ generated from the charge recombination between $\mathbf{P n} \mathbf{Q}^{\bullet+}$ and PnQ ${ }^{\bullet-}$.

Acknowledgment. We thank the members of the Radiation Laboratory of SANKEN, Osaka University, for running the linear accelerator. This work has been partly supported by a Grant-in-Aid for Scientific Research on Priority Area (417), 21st Century COE Research, and others from the Ministry of Education, Culture, Sports, Science and Technology (MEXT) of the Japanese Government.

Supporting Information Available: Ground-state absorption spectra of $\mathbf{P n Q}$ in Bz, transient absorption spectra observed during the pulse radiolysis of $\mathbf{P n Q}(5.0 \mathrm{mM})$ in Ar-saturated $\mathrm{Bz}, \mathrm{DCE}$, and DMF, and transient absorption and emission spectra observed during the laser flash photolysis of PnQ (20$30 \mu \mathrm{M})$ in Ar- and air-saturated Bz. This material is available free of charge via the Internet at http://pubs.acs.org.

\section{References and Notes}

(1) Mozumder, A.; Hatano, Y. Charged Particle and Photon Interactions with Matter: Chemical, Physicochemical, and Biological Consequences with Applications; Marcel Dekker Inc.: NewYork, 2004.

(2) (a) Shida, T.; Hamill, W. H. J. Chem. Phys. 1966, 44, 2369, 2375, 3472. (b) Shida, T.; Kato, T. Chem. Phys. Lett. 1979, 68, 106. (c) Grimison, A.; Simpson, G. A. J. Phys. Chem. 1968, 72, 1776. (d) Ishida, A.; Fukui, M.; Ogawa, H.; Tojo, S.; Majima, T.; Takamuku, S. J. Phys. Chem. 1995, 99, 10808. (e) Majima, T.; Tojo, S.; Ishida, A.; Takamuku, S. J. Org. Chem 1996, 61, 7793. (f) Majima, T.; Tojo, S.; Ishida, A.; Takamuku, S. J. Phys. Chem. 1996, 100, 13615.

(3) (a) Honda, E.; Tokuda, M.; Yoshida, H.; Ogasawara, M. Bull. Chem. Soc. Jpn. 1987, 60, 851. (b) Huddleston, R. K.; Miller, J. R. J. Phys. Chem. 1982, 86, 2410. (c) Majima, T.; Fukui, M.; Ishida, A.; Takamuku, S. J. Phys. Chem. 1996, 100, 8913. (d) Majima T.; Tojo, S.; Takamuku, S. J. Phys. Chem. A 1997, 101, 1048.

(4) (a) Candeias, L. P.; Wilderman, J.; Hadziioannou, G.; Warman, J. M. J. Phys. Chem. B 2000, 104, 8366. (b) Candeias, L. P.; Grozema, F. C. Padmanaban, G.; Ramakrishnan, S.; Siebbeles, L. D. A.; Warman, J. M. J. Phys. Chem. B 2003, 107, 1554. (c) Priyadarsini, K. I.; Mohan H.; Birkett, P. R.; Mittal, J. P. J. Phys. Chem. 1996, 100, 501. (d) Seki, S.; Koizumi, Y.; Kawaguchi, T.; Habara, H.; Tagawa, S. J. Am. Chem. Soc. 2004, 126, 3521. (e) Grozema, F. C.; Hoofman, R. J. O. M.; Candeias, L. P.; de Haas, M. P.; Warman, J. M.; Siebbeles, L. D. A. J. Phys. Chem. A 2003, 107, 5976.

(5) (a) Faulkner, R.; Bard, A. J. Electroanal. Chem. 1977, 10, 1-95. (b) Bard, A. J.; Faulkner, L. R. Electrochemical Methods, Fundamentals and Applications; 2nd ed.; Wiley: New York, 2001; pp 736-745. (c) Richter, M. M. Chem. Rev. 2004, 104, 3003.

(6) (a) Oyama, M.; Okazaki, S. Anal. Chem. 1998, 70, 5079. (b) Lai, R. Y.; Fleming, J. J.; Merner, B. L.; Vermeij, R. J.; Bodwell, G. J.; Bard, A. J. J. Phys. Chem. A 2004, 108, 376. (c) Prieto, I.; Teetsov, J.; Fox, M. A.; Vanden Bout, D. A.; Bard, A. J. J. Phys. Chem. A 2001, 105, 520. (d) Chandross, E. A.; Longworth, J. W.; Visco, R. E. J. Am. Chem. Soc. 1965, 87, 3259. (e) Chandross, E. A.; Dempster, C. J. J. Am. Chem. Soc. 1970, 92, 3586. (f) Maloy, J. T.; Bard, A. J. J. Am. Chem. Soc. 1971, 93, 5968. (g) Faulkner, L. R.; Tachikawa, H.; Bard, A. J. J. Am. Chem. Soc. 1972, 94,691 .

(7) (a) Odom, S. A.; Parkin, S. R.; Anthony, J. E. Org. Lett. 2003, 5 , 4245. (b) Lavastre, O.; Illitchev, I.; Jegou, G.; Dixneuf, P. H. J. Am. Chem. 
Soc. 2002, 124, 5278. (c) Harriman, A.; Zeissel, R. Coord. Chem. Rev. 1998, 171, 331. (d) Kushmerick, J. G.; Holt, D. B.; Pollack, S. K.; Ratner, M. A.; Yang, J. C.; Schull, T. L.; Naciri, J.; Moore, M. H.; Shashidhar, R. J. Am. Chem. Soc. 2002, 124, 10654. (e) Galoppini, E.; Guo, W.; Zhang, W.; Hoertz, P. G.; Qu, P.; Meyer, G. J. J. Am. Chem. Soc. 2002, 124, 7801.

(f) Hortholary, C.; Coudret, C. J. Org. Chem. 2003, 68, 2167. (g) Huang, S.; Tour, J. M. J. Am. Chem. Soc. 1999, 121, 4908.

(8) (a) Elangovan, A.; Chen, T.-Y.; Chen, C.-Y.; Ho, T.-I. Chem. Commun. 2003, 2146. (b) Elangovan, A.; Yang, S.-W.; Lin, J.-H.; Kao, K.-M.; Ho, T.-I. Org. Biomol. Chem. 2004, 2, 1597.

(9) Stephens, R. D.; Castro, C. E. J. Org. Chem. 1963, 28, 3313.

(10) (a) Kawai, K.; Yoshida, H.; Takada, T.; Tojo, S.; Majima, T. J. Phys. Chem. B 2004, 108, 13547. (b) Takada, T.; Kawai, K.; Tojo, S.; Majima, T. J. Phys. Chem. B 2003, 107, 14052. (c) Hara, M.; Tojo, S.; Majima, T. Chem. Phys. Lett. 2004, 387, 283.

(11) Saltiel, J.; Megarity, E. D. J. Am. Chem. Soc. 1972, 94, 2742.

(12) Faulkner, L. R.; Tachikawa, H.; Bard, A. J. J. Am. Chem. Soc. 1972, 94, 691.
(13) (a) Millefiori, S. J. Heterocycl. Chem. 1970, 7, 145. (b) Marcoux, L.; Adams, R. N. J. Electroanal. Chem. 1974, 49, 111. (c) Wawzonek, S.; Wearring, D. J. Am. Chem. Soc. 1959, 81, 2067. (d) Katz, M.; Riemenschneider, P.; Wendt, H. Electrochim. Acta 1972, 17, 1895. (e) Zhang, Y.; Qian, S.-P.; Fun, H.-K.; Xu, J.-H. Tetrohedron Lett. 2000, 41, 8141. (f) Sioda, R. E.; Cowan, D. O.; Koski, W. S. J. Am. Chem. Soc. 1967, 89, 230.

(14) (a) Mohan, H.; Mittal, J. P. J. Phys. Chem. A 1999, 103, 379. (b) Carmichael, I.; Helman, W. P. J. Phys. Chem. Ref. Data 1987, 16, 239.

(15) (a) Liu, D. K. K.; Faulkner, L. R. J. Am. Chem. Soc. 1977, 99, 4594. (b) Bohne, C.; Abuin, E. B.; Scaiano, J. C. J. Am. Chem. Soc. 1990 $112,4226$.

(16) (a) Eberson, L. J. Am. Chem. Soc. 1967, 89, 4669. (b) Neikam, W. C.; Desmond, M. M. J. Am. Chem. Soc. 1964, 86, 4811. (c) Eberson, L. J. Am. Chem. Soc. 1967, 89, 4669. (d) Dileesh, S.; Gopidas, K. R. J. Photochem. Photobiol. A 2004, 162, 115.

(17) Fungo, F.; Wong, K.-T.; Ku, S.-Y.; Hung, Y.-Y.; Bard, A. J. J. Phys. Chem. B 2005, 109, 3984. 